\title{
Cortical Blindness During Coil Embolization of an Unruptured Intracranial Aneurysm
}

\author{
-Case Report-
}

\author{
Jun SHINODA, Yasuhiko AJImI, Motoyuki YAmAdA, and Satoshi ONOZUKA*
}

Department of Neurosurgery, Shizuoka Red Cross Hospital, Shizuoka;

${ }^{*}$ Department of Neurosurgery, Keio University School of Medicine, Tokyo

\begin{abstract}
A 62-year-old woman suffered transient cortical blindness during coil embolization for a saccular aneurysm in the posterior circulation, with cerebral angiography using non-ionic contrast material. Just before completion of the procedure, the patient showed abrupt onset of restlessness and blindness. At this point $150 \mathrm{ml}$ of contrast material had been injected into the left vertebral artery. Immediate computed tomography showed persistence of contrast medium in the bilateral occipital lobes and part of the left frontal lobe. Repeat angiography showed no evidence of embolism. Single photon emission computed tomography obtained the next day showed decreased perfusion in the bilateral occipital lobes. Magnetic resonance imaging on the third day showed multiple lesions in the bilateral occipital lobes. Her vision was restored gradually. Transient cortical blindness may be associated with osmotic disruption of the blood-brain barrier in the bilateral occipital lobes. Endovascular neurosurgeons must be aware of this rare complication.
\end{abstract}

Key words: cortical blindness, coil embolization, intracranial aneurysm, occipital lobe

\section{Introduction}

Transient cortical blindness is well known as a rare, dramatic, but self-limiting complication of cerebral angiography. ${ }^{5)}$ Transient cortical blindness is associated with transient reversible damage of the blood-brain barrier (BBB) of the bilateral occipital lobes by contrast medium, but is considered unusual with non-ionic contrast materials. Endovascular neurosurgery is used to treat an increasing number of patients because of the minimum invasiveness, so endovascular neurosurgeons must be aware of this rare complication. Here we report a case of transient cortical blindness during embolization of an unruptured intracranial aneurysm of the posterior circulation using Guglielmi detachable coils (GDCs).

\section{Case Report}

A 62-year-old woman presented to our outpatient department with a complaint of heavy feeling in the left temporal region. She had no past history of

Received January 13, 2004; Accepted March 23, 2004 allergy or intoxication. Magnetic resonance (MR) angiography demonstrated multiple intracranial aneurysms. On her first admission to our hospital, cerebral angiography was performed using $80 \mathrm{ml}$ of non-ionic contrast material ioversol for definite diagnosis. Angiography revealed intracranial aneurysms at the left vertebral artery (VA)-posterior inferior cerebellar artery (PICA) junction, $4 \times 4 \mathrm{~mm}$ in diameter, and at the bifurcation of the left middle cerebral artery (MCA). She was discharged with no neurological complication on the following day.

One month later, she was admitted again for endovascular treatment of the left VA-PICA aneurysm. Preoperative left vertebral angiography was used to image the aneurysm in detail again (Fig. 1A). Embolization was performed safely in about 3 hours with complete obliteration of aneurysm using four GDCs and the same contrast medium. However, just before completion of the procedure, when contrast material was injected into left internal carotid artery, the patient suffered sudden onset of loss of vision and restlessness. At this point $150 \mathrm{ml}$ of contrast material had been injected into the left VA. Computed tomography (CT) was performed immediately, which showed the presence 


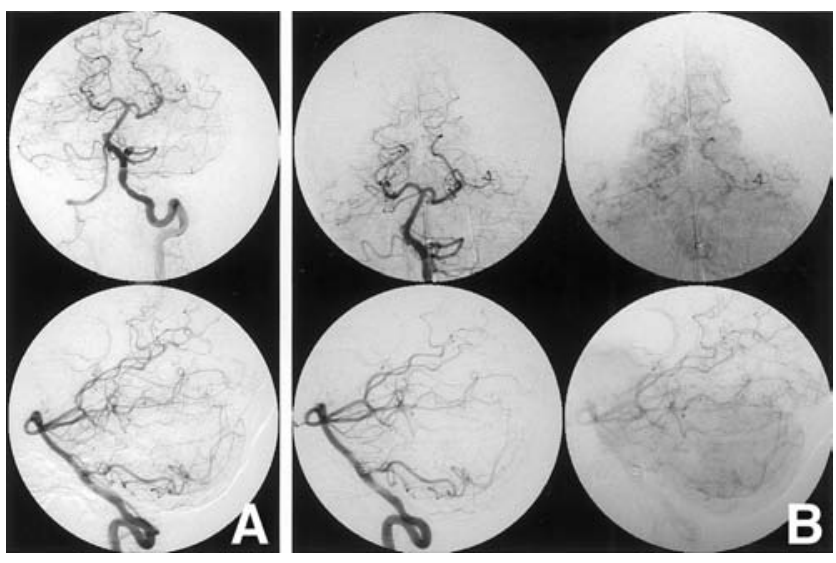

Fig. 1 Left vertebral angiograms before (A) and after (B) endovascular treatment of a saccular aneurysm at the left vertebral arteryposterior inferior cerebellar artery junction, $4 \times 4 \mathrm{~mm}$ in diameter. The patient complained of complete blindness just before the latter angiogram. Note that there is no evidence for normal artery embolization after the procedure.

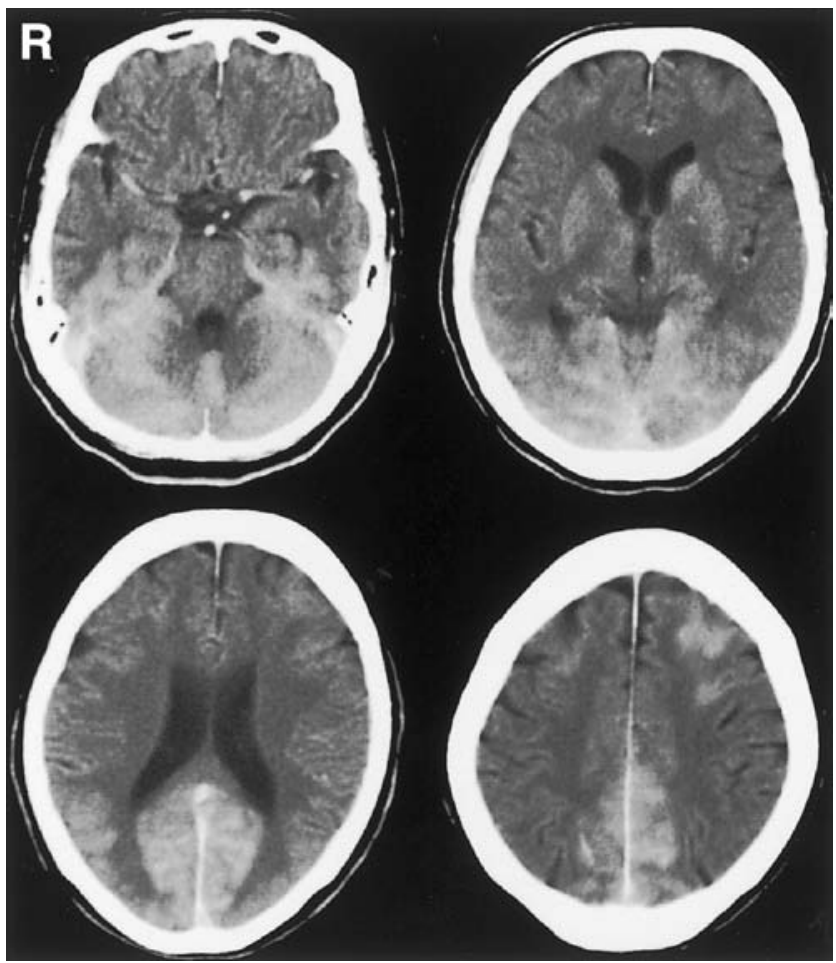

Fig. 2 Computed tomography scans immediately after the patient's complaint of blindness, demonstrating significant contrast enhancement of the bilateral occipital and temporal lobes, and part of the left frontal lobe.

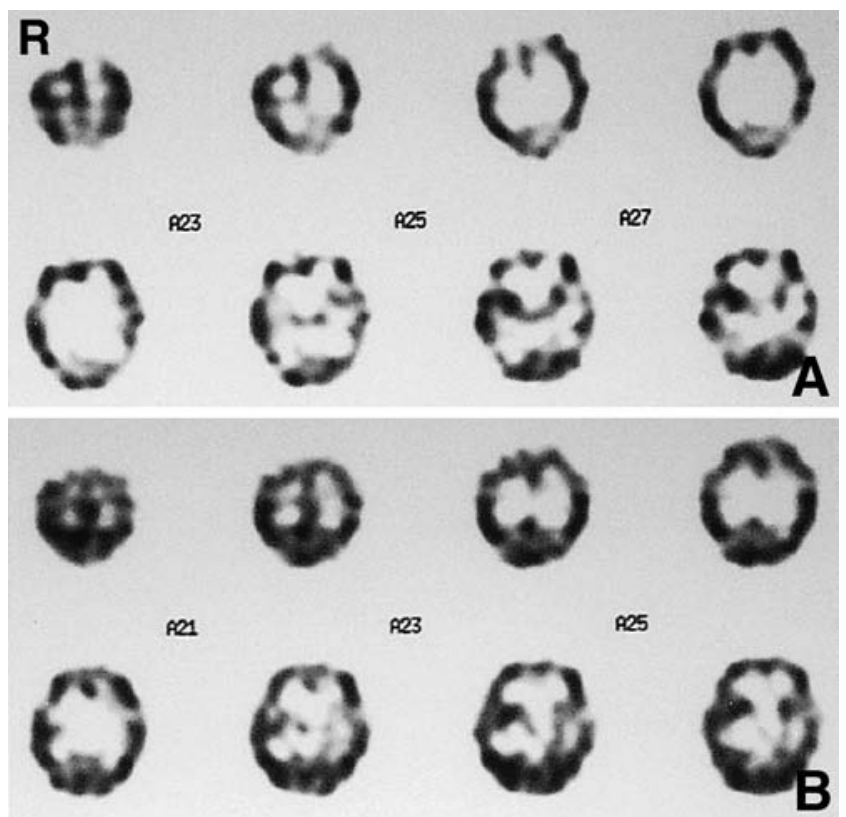

Fig. 3 Single photon emission computed tomography scans using ${ }^{99 m}$ Tc-hexamethylpropylene amine oxide on the second day (A) and 17th day (B) after embolization. Cerebral perfusion transiently decreased in the bilateral occipital lobes and posterior part of the bilateral temporal lobes, but had improved on the 17th day.

of contrast medium in the bilateral occipital lobes and part of the left frontal lobe (Fig. 2). She recovered her vision within one hour with conservative therapy. However, subsequent vertebral angiography again caused the complaint of total blindness. Repeat angiography showed no evidence for arterial embolism (Fig. 1B).

After the procedure, she was treated conservatively with hydration and administration of steroids. Single photon emission computed tomography (SPECT) with $99 \mathrm{~m} \mathrm{Tc}$-hexamethylpropylene amine oxide (HMPAO) the next day showed decreased perfusion in the bilateral occipital lobes (Fig. 3A). MR imaging on the third day showed multiple lesions in the bilateral occipital and parietal lobes (Fig. 4A).

The patient regained vision gradually. On the 15th day, examination detected incomplete left lower quadrantanopsia and another irregular deficit on the right. ${ }^{99 \mathrm{~m}} \mathrm{Tc}-\mathrm{HMPAO}$ SPECT on the $17 \mathrm{th}$ day demonstrated improvement of cerebral perfusion in the bilateral occipital lobes (Fig. 3B). MR imaging with gadolinium enhancement on the same day revealed significant reduction of the enhanced lesions, which were limited to the bilateral occipital 

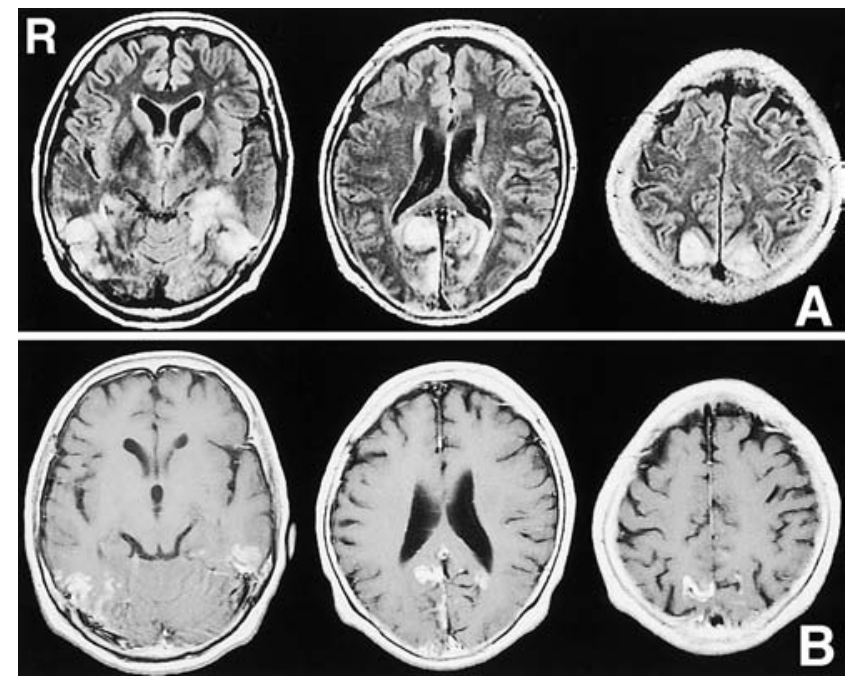

Fig. 4 Magnetic resonance images obtained on the third day (A: fluid-attenuated inversion recovery images) and $17^{\text {th }}$ day $\left(B: T_{1^{-}}\right.$ weighted images with gadolinium enhancement) showing lesions in the bilateral occipital lobes. Most of the lesions had disappeared on the 17 th day, but several lesions remained enhanced within the cerebral cortex.

and parietal cortices (Fig. 4B). Three weeks after embolization, the patient was discharged with normal visual acuity, but the deficit persisted in the visual field. Two months later she underwent successful clipping of the left MCA aneurysm.

\section{Discussion}

Transient cortical blindness associated with nonionic contrast material is recognized as a rare complication of cerebral/cardiac/brachial angiography, myelography, and aortography..$^{1,8,10,14-16,19)}$ The incidence of transient cortical blindness is as high as $1 \%$ with cerebral angiography. ${ }^{5,17)}$ However, the recent introduction of non-ionic contrast materials is considered to have significantly reduced the risk, and this complication has become extremely rare.

The clinical features of cortical blindness are loss of bilateral visual perception which may be denied by the patient, normal optic fundi, normal light reflex, and preservation of ocular motility. ${ }^{18)}$ Transient cortical blindness associated with contrast material occurs within minutes to 6 hours after angiography. The duration of symptoms varies from a few hours to several days. ${ }^{1,8,9,14,15)}$ Therefore, transient cortical blindness may go undiscovered if resolution is rapid and the patient denies any visual symptoms. ${ }^{9)}$

Transient cortical blindness is thought to occur when an adverse reaction to the contrast material results in osmotic disruption of the BBB.7,12) Vasospasm, micro-embolism, and immunological events have also been suggested as mechanisms. ${ }^{2,5,6,12,16,17)}$ The strength of the BBB is known to vary within the central nervous system. The occipital cortex is considered to be the most vulnerable region, ${ }^{4)}$ because the BBB over the visual cortex is believed to be incomplete. ${ }^{17)}$ However, the exact mechanism of neurotoxicity associated with contrast agents is still speculative. Brain CT in the acute stage shows enhancement of the bilateral occipital lobes, which clears within several hours. ${ }^{12,17,19)}$ Limiting the amount of dye is strongly recommended to minimize the risk of transient cortical blindness. ${ }^{15)}$ Hydration and intravenous administration of steroids are preferred as the initial treatment despite the absence of clinical evidence. ${ }^{3,12)}$

In our case, CT performed immediately after the endovascular procedure showed persistence of contrast material in the bilateral occipital lobes and the left frontal lobe. The SPECT findings of transient cortical blindness as a complication of angiography are not well characterized. In a similar case to ours, ${ }^{99 m}$ Tc-HMPAO SPECT on the following day revealed decreased perfusion in the bilateral occipital regions. ${ }^{11)}$ These results strongly suggest that transient cortical blindness is associated with acute phase SPECT evidence of reduction of perfusion in the bilateral occipital lobes. The SPECT findings on the 17th day suggested that the reduction of occipital perfusion is actually transient and reversible. At present no conclusive evidence has been established about the SPECT characteristics of transient cortical blindness induced by contrast medium.

Fluid-attenuated inversion recovery MR imaging obtained after angiography depicted multiple high intensity lesions. The greater part of the MR imaging lesions had resolved within 16 days of the onset, but the persisting lesions were still enhanced by gadolinium contrast material on the 17th day. We speculate that the former lesions could be categorized as classical transient cortical blindness in which the $\mathrm{BBB}$ is temporally malfunctioning due to long-term exposure to contrast medium, ${ }^{13)}$ whereas in the latter lesions represented irreversible disruption of the BBB and neuronal elements. We assume that the osmotic effect of the contrast material was responsible for the disruption of the $\mathrm{BBB}$, whereas the toxicity of the contrast medium caused irreversible damage to the BBB and/or underlying neuronal elements. Vertebral angiography immediately after the onset of visual symptoms showed no evidence of 
microembolization or infarction, but these factors cannot be totally excluded.

The recent trend for less invasive surgery and use of GDCs has promoted the use of endovascular neurosurgery. Nowadays, many neurosurgeons prefer embolization to direct clipping surgery for intracranial aneurysms, especially in the posterior circulation. However, endovascular neurosurgeons need to be aware that repeat vertebral angiography carries the risk of transient cortical blindness, as a larger number of patients are exposed to the risk of neurological complications including transient cortical blindness.

\section{References}

1) Antonellis J, Kostopoulos $K$, Rambouni A, Margaris N, Kranidis A, Salahas A, Ifantis G, Koroxenidis G: Cortical blindness following coronary angiography: a rare but self-cured complication, two case reports. Angiology 47: 803-806, 1996

2) Demirtas M, Birand A, Usal A: Transient cortical blindness after second coronary angiography: Is immunological mechanism possible? Cathet Cardiovasc Diagn 31: 16, 1994

3) Helsley JD: Cortical blindness following cerebral angiography. W V Med J 91: 324, 1995

4) Henzlova MJ, Coghlan HC, Dean LS, Taylor JL: Cortical blindness after left internal mammary artery to left anterior descending coronary artery graft angiography. Cathet Cardiovasc Diagn 15: 37-39, 1988

5) Horwitz NH, Wener L: Temporary cortical blindness following angiography. J Neurosurg 40: 583-586, 1974

6) Jackson A, Stewart G, Wood A, Gillespire JE: Transient global amnesia and cortical blindness after vertebral angiography: further evidence for the role of arterial spasm. AJNR Am J Neuroradiol 16: 955-959, 1995

7) Junck L, Marshall WH: Neurotoxicity of radiological contrast agent. Ann Neurol 13: 469-484, 1983

8) Katayama J, Fukami K, Yoshida H, Mizunuma Y, Moriai N, Takino T, Hosokawa S, Hashimoto K, Nakai K, Kawazoe K, Hiramori K: Transient cortical blindness following bypass graft angiography.
Angiology 46: 937-946, 1995

9) Kermode AG, Chakera T, Mastaglia FL: Low osmolar and non-ionic X-ray contrast media and cortical blindness. Clin Exp Neurol 29: 272-276, 1992

10) Kinn RM, Breisblatt WM: Cortical blindness after coronary angiography. Cathet Cardiovasc Diagn 22: 177-191, 1991

11) Kodama $Y$, Yasui $T$, Kageyama $Y$, Ichikawa K: [Transient cortical blindness after cerebral angiography using non-ionic contrast media]. Shinkei Naika 50: 357-360, 1999 (Jpn, with Eng abstract)

12) Lantos G: Cortical blindness due to osmotic disruption of the blood-brain barrier by angiographic contrast material: CT and MRI studies. Neurology 39: 567-571, 1989

13) Nakai Y, Hyodo A, Okazaki M, Shibata Y, Matsumaru Y, Nose T: [Transient cortical blindness and convulsion mimicking a hemorrhagic complication during embolization of the cerebellar AVM]. No Shinkei Geka 27: 249-253, 1999 (Jpn, with Eng abstract)

14) Parry R, Rees JR, Wilde P: Transient cortical blindness after coronary angiography. Br Heart J 70: 563-564, 1993

15) Rama BN, Pagano TV, DelCore M, Knobel KR, Lee J: Cortical blindness after catheterization: effect of rechallenge with dye. Cathet Cardiovasc Diagn 28: 149-151, 1993

16) Skinner JS, Jackson MJ, Gholkar A, Adams PC: Cortical blindness during left internal mammary angiography. Int J Cardiol 52: 119-123, 1995

17) Studdard WE, Davis DO, Young SW: Cortical blindness after cerebral angiography. J Neurosurg 54: 240-244, 1981

18) Upson JE, Mccarie M: Transient posttraumatic cortical blindness: Case report. Milit Med 149: 31-32, 1984

19) Utz R, Ekholm SE, Isaac L, Sands M, Fonte D: Local blood-brain barrier penetration following systemic contrast medium administration. Acta Radiol 29: 237-242, 1988

Address reprint requests to: J. Shinoda, M.D., Department of Neurosurgery, Shizuoka Red Cross Hospital, 8-2 Oute-machi, Shizuoka, Shizuoka 420-0853, Japan. e-mail:shinodaj@mbd.nifty.com 